\title{
The Road to Conductors of High Temperature Superconductors: 10 Years do make a Difference!
}

\author{
David C Larbalestier \\ Department of Materials Science and Engineering, Department of Physics and the Applied Superconductivity Center \\ University of Wisconsin, Madison WI 53706 USA
}

\begin{abstract}
Amongst all the predictions for a superconducting future that were made in the early days after the discovery of high temperature superconductivity, most depended on the development of conductors from which magnets, motors, power cables etc. could be constructed. Until 1989, there seemed little prospect of such a technology because polycrystalline forms of HTS were granular and transmitted current very poorly. Starting with an empirical breakthrough in 1989, a strong set of large scale demonstration devices have been made with high temperature superconductors. The foundation of all this technology lies in the conductor. For HTS conductors, 10 years does indeed make a difference, as this review summarizes.
\end{abstract}

\section{THE NEW SUPERCONDUCTING AGE: 1987}

The proceedings of the Applied Superconductivity Conferences since 1986 make fascinating reading [1] for those interested in the progress of our field. 1986 was a year of retrospectives, as befits the $75^{\text {th }}$ anniversary of the discovery of superconductivity. Three features of the retrospective talks given at that meeting struck me. Discussion of the construction of various superconducting magnets using cold worked wires [2] having modest superconducting properties showed how empiricism had inched superconductivity into a technology, all the while fighting an entrenched hypothesis - the sponge model - that was quite wrong. Particularly fascinating to me was the review by Berlincourt [3] of the long road to understanding that there were in fact two types of superconductivity and the way in which their definitive discovery in $\mathrm{Pb}$ alloys in the 1930s by Shubnikov (part at least of this work was published in Nature [4]) was "lost" when he was imprisoned and then killed. Not until some 25 years later did indubitable experiment with $\mathrm{Nb}_{3} \mathrm{Sn}$ force a complete reexamination of experiment (also the forcing recognition of the "theoretical" discovery of extreme type II superconductivity by Abrikosov in 1952 [3]). Type II superconductivity was then recognized as a "bulk", not just a filamentary, phase and the first era of superconducting applications got under way in 1962. But exciting as all this was, one of the most striking features of the retrospective was the absence of discussion about higher transition temperatures than the $23 \mathrm{~K}$ of $\mathrm{Nb}_{3} \mathrm{Ge}$. A lonely paper published about this time by DewHughes [6] discussed the prospects of raising $\mathrm{T}_{\mathrm{c}}$ of $\mathrm{Nb}_{3} \mathrm{Si}$ to about $30 \mathrm{~K}$ by exotic processing. Sadly, Berndt Matthias, the great discoverer of new materials, had died only a few months before ASC86. It is probably true that not one atten-

Manuscript received August 27, 1996.

This work is supported by DARPA, DOE, EPRI, and NSF. dee at the conference knew anything of the paper of Bednorz and Muller [7] on possible superconductivity in the La-Ba$\mathrm{Cu}-\mathrm{O}$ system that was slowly wending its way to publication right after ASC86 finished. The delicious irony of this strange conjunction, 75 years of retrospective, indeed rather valedictory musings conducted in total ignorance of the already type-set, unthought-of breakthrough, has enlivened many a subsequent after-hours conversation amongst those who were present at this last pre-HTS ASC conference.

All was changed in 1988! One of the most remarkable aspects of the change is that it was almost completely binary. The key change occurred between December 1986 and March 1987, starting with confirmation of the IBM-Zurich report of $T_{c}$ values above $30 \mathrm{~K}$ by the groups of Chu and of Kitazawa at the MRS meeting in November 1986. However, the real transformation occurred with the announcement of superconductivity above $77 \mathrm{~K}$ in February 1987 [8]. Suddenly all of Kamerlingh Onnes' 1913 dreams [9] seemed possible.

It is almost certainly true that no one who was at the heart of the exciting times of 1987 can recall a scientific experience that is even remotely comparable. Headlines in every conceivable popular newspaper (not just the New York Times, journal of record though some seemed to think it had become!), magazine (Time, Fortune, Popular Mechanics etc.) led to a vast public interest in superconductivity. Many thought (and still think) of superconductivity as being so fascinating that it is almost magical. Stimulated by the heady excitement of the times and the unusual attention lavished on the field by the press, there was no shortage of comments, most conveying the excitement that we all felt. Unfortunately, most of the cautionary words were filtered out.

Of course public interest led to institutional and political interest. The National Academy of Sciences asked John Hulm to convene a panel and to report on the applications potential of high temperature superconductors. Strong argument ensued. Was the discovery so monumental that roomtemperature-superconductors had already been discovered? Could anyone raised in the restricted temperature space of the low temperature superconductors (LTS) possibly comprehend the immense potential of HTS? The flavor of the report [10] was passionately discussed. In my view, the result, though too cautious for some, still reads remarkably well and the balance between optimism and caution seems entirely appropriate. Perhaps because of its perceived caution - and perhaps also because of the Iran-Contra hearings - the White House decided to hold a Presidential Conference on Super- 
conductivity [11] in summer of 1987 . Who can forget the spectacle of the President and all his cabinet devoting their attention to the field of superconductivity in a meeting explicitly designed to exclude scientists from outside the US at a time when things were so fluid and so uncertain? Still, the spirit of excitement engendered by the times stimulated new entrepreneurs to enter the field and awakened this spirit in some already in the field. American Superconductor Corporation, Conductus, Illinois Superconductor Incorporated, and Superconducting Technologies Incorporated are just a few of the companies that reflect this spirit. We can now celebrate their transition to companies employing several hundred people seeking to commercialize HTS.

A final complication in all of this was the Superconducting Super Collider (SSC). Unfortunately, just as the largest superconducting project in the world was approved, the discovery of HTS provided a focus for its re-evaluation. The view gathered ground in a few quarters that the SSC magnets could in fact be made from HTS. An extreme manifestation of this view was that the SSC could only be justified if it was built from HTS materials. Unfortunately accelerator magnets are amongst the most complex of all. They require conductors with many thousand, fine $(<10 \mu \mathrm{m})$ filaments, very high overall $J_{c}$, high strength and strain tolerance, multi-kiloamp cable forms, all fabricated at reasonable cost. As a special DOE-Basic Energy Sciences panel concluded [12] late in 1987, HTS materials were quite unable to supply these properties, a statement that is still true today. In fact one of the important themes of this review and that of Paul Grant [13] is that HTS materials have so far not attacked the markets of LTS at all. Rather they have expanded the domain of superconductivity into new fields where the refrigeration complexities or costs of LTS materials make superconductivity economically unattractive.

In keeping with the thrust of my comments in this first section, my paper is exclusively devoted to materials issues and to factors relevant to large scale applications of HTS technology. Paul Grant [13] amplifies some of my territory, while Paul Chu [14] discusses the materials discovery aspects of high temperature superconductivity (HTS) and Ted Van Duzer [15] the electronic applications of HTS. I will concentrate on the way that the very complex materials science of the cuprates impeded the fabrication of the conductors needed to implement the technological dreams that sprouted virtually everywhere in the heady days of 1987.

\section{CONDUCTORS DETERMINE THE TECHNOLOGY}

The vast publicity of 1987 led many to the conclusion that we were about to enter a superconducting age. This thought was greatly amplified by anticipation that room temperature superconductivity was just around the corner. But in fact, as noted in the National Academy report [10], the key factor for technology is the critical current density, $\mathrm{J}_{\mathrm{c}}$, rather than the transition temperature, $T_{c}$. This fact alone meant that con- ductors made from LTS superconductors could not just be discarded, since those made from $\mathrm{Nb}-\mathrm{Ti}$ and $\mathrm{Nb}_{3} \mathrm{Sn}$ were by now very sophisticated and had formed the basis of a large and sophisticated magnet technology responsible for a large medical therapeutic technology, Magnetic Resonance Imaging (MRI), for the multi-kilometer circumference accelerators at Fermilab and at DESY, and for many diverse specialty magnets used for a very broad range of applications. But in fact, with the number of scientists studying superconductivity increasing by more than an order of magnitude in less than a year, the experience base of those actually making a living from the low temperature superconductors (LTS) often seemed to be completely neglected or considered trivial. As a result 1987 was not the time when the strong materials and physics challenges posed by HTS raised widespread concern. However, this inattention was repaid by 3 or 4 years of caution, even gloom, from 1988 on. The truly remarkable message of today is that a viable large scale HTS technology seems assured due to the remarkable advances of the last 5 years. A very striking characteristic for me is that most of these advances have come from belief and inspired empiricism. Only recently has the science underpinning the processing of HTS materials into useful conductor forms become significant. This is surely a very important guide to the future potential of HTS materials. In the rest of this paper, I hope to sketch out the basic elements that have driven the development of HTS conductors in the last 5 or 6 years since the realization that Heine, Tenbrink, and Thoener [16] had found a way to process the $\mathrm{Bi}_{2} \mathrm{Sr}_{2} \mathrm{CaCu}_{2} \mathrm{O}_{\mathrm{x}}$ compound in such a way that reasonably high critical current densities $\left(\mathrm{J}_{\mathrm{c}}\right)$ could be transmitted in genuinely polycrystalline and imperfectly aligned wire forms.

\section{LTS CONDUCTORS MOVE THE BAR}

What is the situation of HTS conductors today? To start with proponents of HTS conductors must recognize that LTS conductors have not stood still, either in properties or in their cost and reliability of production and application [17]. In fact one of the signs of the maturing of HTS conductor is that cost figures are beginning to appear in public discussion. The lessons of LTS are in some respects also valid for HTS, for they show the benefit of continuously investing in R\&D. A vital characteristic of LTS development has been that there has been a continuous developmental interaction [18] between the user community (which includes both the commercially driven need to reduce the conductor cost, and the highperformance community (e.g. ITER [19]) who continuously need to push conductors into new performance territory, cost being a secondary issue), the wire producers, and the materials understanding community. This has produced a very tightly interwoven national laboratory-industry-university collaborative community that has facilitated and made enormous breakthroughs in performance and in cost-benefit ratio over the last 15 years [18]. This is illustrated in Fig. 1 for 


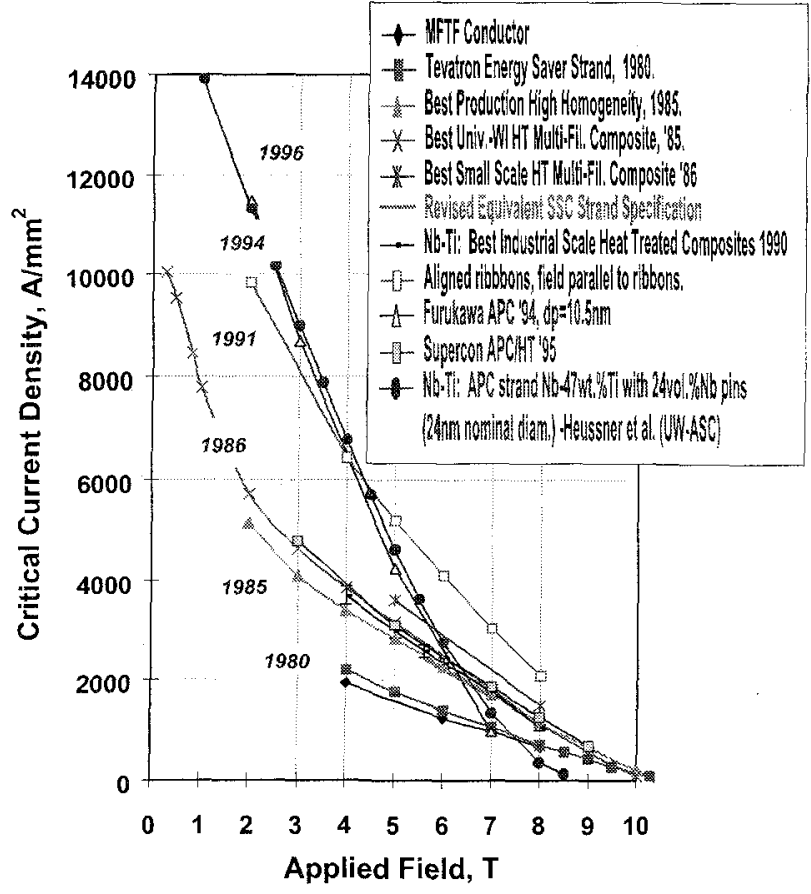

Figure 1: Evolution of critical current density in conductor-like forms of $\mathrm{Nb}$ Ti in recent years (compilation courtesy of Peter Lee, UW).

$\mathrm{Nb}$ - $\mathrm{Ti}$, surely the simplest of all practical superconductors. Even so, the basic flux pinning mechanism is still not clear in this material, as several papers in this conference attest, nor is the flux pinning mechanism yet fully optimized [20]. The sophistication of present conductors of $\mathrm{Nb}-\mathrm{Ti}$ and $\mathrm{Nb}_{3} \mathrm{Sn}$ is illustrated in Figure 2. One of the basic issues of the next 5 years is whether these will be replaced by HTS conductors or whether HTS conductors will develop their own markets, leaving LTS conductors in production.

\section{DEFINING A USEFUL CONDUCTOR}

If the above comments about LTS conductors are correct, what is it that defines a useful conductor? The most basic requirement of any conductor is that it carry a high critical current density. Exactly what defines high is not precisely defined. The best present $(\mathrm{Bi}, \mathrm{Pb})_{2} \mathrm{Sr}_{2} \mathrm{Ca}_{2} \mathrm{Cu}_{3} \mathrm{O}_{x}$ conductors have supercurrent densities at $77 \mathrm{~K}$, self-field $(77 \mathrm{~K}, 0 \mathrm{~T})$ of $20-50 \mathrm{kA} / \mathrm{cm}^{2}$, depending on the length and sophistication of the conductor. $J_{c}(4 \mathrm{~K}, 0 \mathrm{~T})$ values are some 5-7 times higher. By comparison Nb-Ti alloys have $\mathrm{J}_{\mathrm{c}}(4 \mathrm{~K}, 5 \mathrm{~T})$ values of 300 $\mathrm{kA} / \mathrm{cm}^{2}$ and $\mathrm{Nb}_{3} \mathrm{Sn}$ conductors $\mathrm{J}_{\mathrm{c}}(4 \mathrm{~K}, 10 \mathrm{~T})$ of $100-200$ $\mathrm{kA} / \mathrm{cm}^{2}$. Thus the useful range for superconductor $J_{c}$ is generally taken to lie in the range $5-250 \mathrm{kA} / \mathrm{cm}^{2}$ in the range of operation. Of course economics exerts a strong role, as is well demonstrated by the influence that the competitive market of MRI has had on the cost and design of simple Nb-Ti conductors for MRI markets. Figure 3 shows a recent 85 filament conductor of $(\mathrm{Bi}, \mathrm{Pb})_{2} \mathrm{Sr}_{2} \mathrm{Ca}_{2} \mathrm{Cu}_{3} \mathrm{O}_{x}$ with rather high $\mathrm{J}_{\mathrm{c}}$. The essential resemblance to Figure 2 is clear, though the contrast of round wire LTS conductor to highly aspected tape

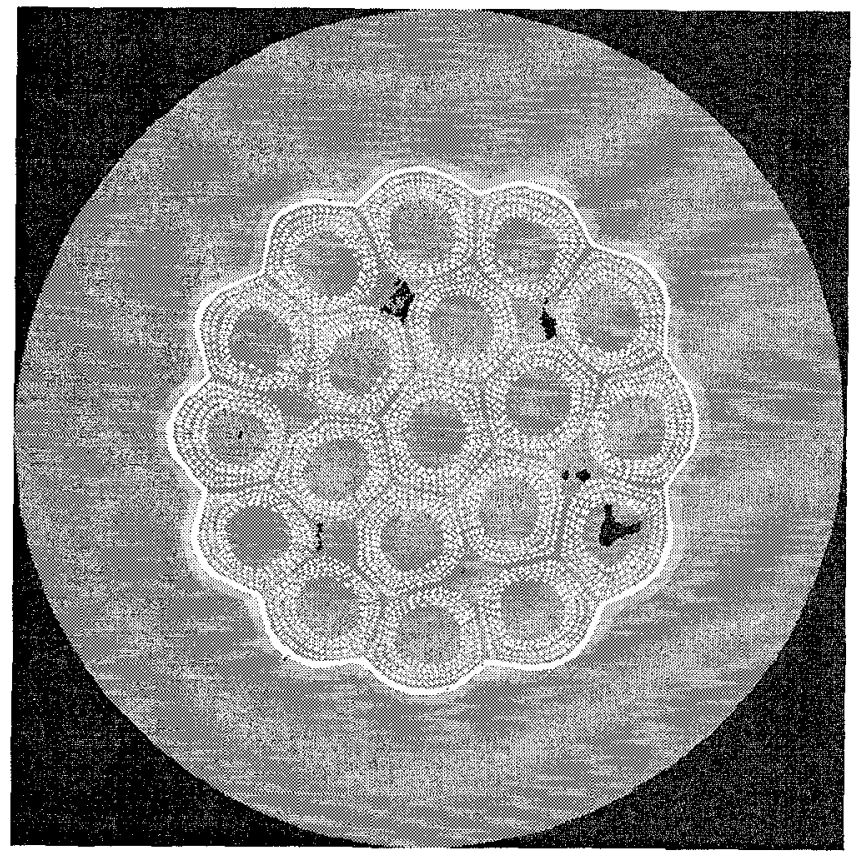

Figure 2: Cross section of a reacted filamentary $\mathrm{Nb}_{3} \mathrm{Sn}$ conductor optimized for high $\mathbf{J}_{\mathrm{c}}$ and low hysteresis loss application for ITER magnets (courtesy E. Gregory, IGC).

for HTS conductor is a vital difference that introduces complications in cabling high amperage conductors from smaller strands having critical currents of 50-100 A.

Also vital for a conductor is a parallel normal conductor to shunt supercurrent around weak spots and to protect the magnet from destruction by burn up during the propagation of normal zones in the conductor. Strain tolerance and strength are vital, both for manufacture, coil winding and to resist electromagnetic stresses during magnet energization. A further vital property is the ability to scale the currentcarrying capacity of the conductor from amps to kiloamps. Magnet protection considerations require that larger magnets require larger current conductors in order to restrain their inductance to manageable values. Finally we note that utility applications are a potentially enormous market for HTS conductors. Many of these require minimized ac losses. Thus the ability to control both intra- and inter-filament hysteresis and eddy current losses is also vital.

The complexity of any real conductor, whether LTS or HTS, is a genuine reason why progress towards sophisticated HTS conductors takes several years. The large amount of development needed to produce internal-tin $\mathrm{Nb}_{3} \mathrm{Sn}$ conductors of high $\mathbf{J}_{\mathrm{c}}$ and low hysteresis loss (see Figure 2) for ITER applications is testimony that this is hard, even for a much better understood LTS conductor with more than 25 years of development behind it.

\section{BSCCO: THE FIRST GENERATION CONDUCTOR}

Conductors of the two higher $\mathrm{T}_{\mathrm{c}}$ BSCCO compounds, $(\mathrm{Bi}, \mathrm{Pb})_{2} \mathrm{Sr}_{2} \mathrm{Ca}_{2} \mathrm{Cu}_{3} \mathrm{O}_{\mathrm{x}}$ (2223) and $\mathrm{Bi}_{2} \mathrm{Sr}_{2} \mathrm{CaCu}_{2} \mathrm{O}_{\times}$(2212) are 


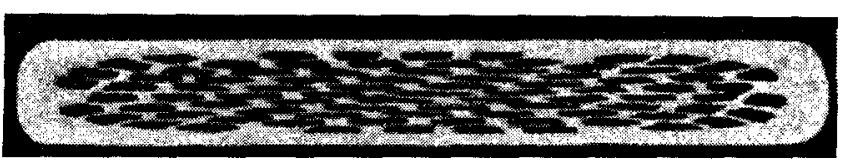

Figure 3: 85 filament BSCCO-2223 conductor (courtesy G. N. Riley Jr., ASC).

now available from several manufacturers. Because of its higher $T_{c}(\sim 107 \mathrm{~K}$ vs. $\sim 90 \mathrm{~K})$, the BSCCO-2223 compound is preferred, since it has $77 \mathrm{~K}$ capability not possessed by BSCCO-2212. However, BSCCO-2212 can be made in round wire form with reasonable $J_{c}$ and its $J_{c}$ values are a little higher than for BSCCO-2223 at $4 \mathrm{~K}$, in spite of its lower $T_{v}$. The better electromagnetic connectivity of BSCCO-2212 is normally taken to explain this apparent anomaly, thus illuminating an important aspect of the challenge of making high $\mathrm{J}_{\mathrm{c}}$, HTS conductors.

The crucial reason why BSCCO conductors have come to market first is because of the weakly bonded, double Bi-O layer that separates the $\mathrm{CuO}_{2}$ planes of strong superconductivity. This layer is decisive for many of its properties. The fact that it is weakly bonded gives it micaceous properties. The easy cleavage between the $\mathrm{Bi}-\mathrm{O}$ layers produces a selfaligning property to the BSCCO grains that permits both deformation and growth texturing of the phase. Both of these factors contribute to enhancing the electromagnetic connectivity of the grains, thus making high transport current densities over long lengths possible. However, the weak bonding of the Bi-O layers also has a very negative effect too, for the significant thickness of the blocking layer $(1.23 \mathrm{~nm})$ decouples neighboring sets of the double or triple $\mathrm{Cu}-\mathrm{O}_{2}$ layers of strong superconductivity from each other, making the two compounds strongly two-dimensional. This weakness of BSCCO is illustrated by Figure 4 which shows the H(T) do-

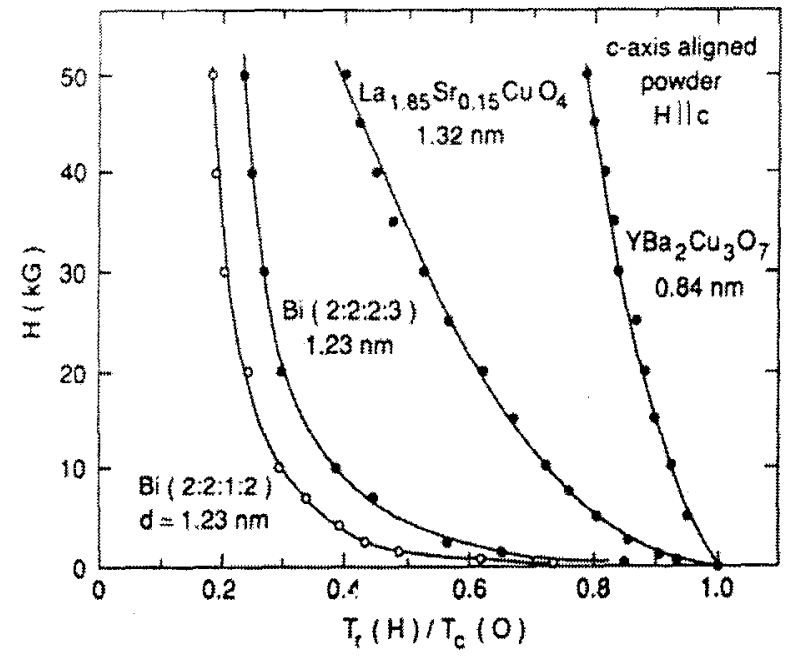

Figure 4: Normalized irreversibility lines for several important HTS. The absolute $T_{c}$ values for the compounds are: Bi-2212 $\sim 90 \mathrm{~K}, \mathrm{Bi}-2223 \sim 107$ $\mathrm{K}, \mathrm{LSCO} \sim 37 \mathrm{~K}, \mathrm{YBCO} \sim 90 \mathrm{~K}$. Finite $\mathrm{J}_{\mathrm{c}}$ is only possible to the left of each line. Dimensions noted by each compound are the blocking layer thickness (Courtesy M. Suenaga ref. 23). main over which a finite $J_{c}$ can be sustained for various HTS compounds. Unlike LTS compounds, whose domain is largely determined by the temperature dependence of the upper critical field $\mathrm{H}_{\mathrm{c} 2}$, the useful domain of HTS is determined by the irreversibility field, $H^{*}(T)$, which can be very much less than the domain defined by $\mathrm{H}_{22}(\mathrm{~T})$.

In spite of these complexities, the early breakthrough of Heine et al. [16] has been very effectively exploited by both industrial and laboratory producers, particularly at Sumitomo Electric Industries in Japan [21] and at American Superconductor Corporation [22] in the USA, both of whom are producing multifilamentary BSCCO-2223 conductors in lengths that now exceed $1 \mathrm{~km}$. It is these conductors that have enabled the prototype motors, power cables, transformers and fault current limiters that are now showing that useful utility devices can be made from HTS conductors. To make these breakthroughs has required a large effort of patient understanding of the current-limiting mechanisms in tapes, a point that we return to in section VII. The work of various members of the wire development group [24] in the US and of the U. of Geneva group [25] has been particularly extensive in this regard.

\section{BIAXIALLY TEXTURED THICK FILMS}

Figure 4 shows that $\mathrm{YBCO}$ has an enormous advantage over $\mathrm{BSCCO}$, so far as the domain of finite $J_{c}$ is concerned. YBCO's problem has been that polycrystalline $J_{c}$ values have been very small unless grown in ways that permit strong biaxial alignment. This techniques was first developed by Iijima et al. [26] using the technique of Ion Beam Assisted Deposition (IBAD). Subsequent refinements [27] of this technique have generated strong programs aimed at making Second-Generation conductors from YBCO, either using the IBAD process or by the Rolling-Assisted Biaxial Texturing (RABiTS) approach [28].

The essence of the structure of such a conductor is shown in Figure 5. Both approaches start with a metallic substrate, a Ni-base alloy substrate for the IBAD process and a pure $\mathrm{Ni}$ substrate for the RABiTS process. The IBAD step occurs during the sputtering of a YSZ template layer on which the YBCO is subsequently deposited. The role of the IBAD process is to align the YSZ in a biaxial fashion on top of the randomly aligned, polycrystalline alloy substrate. Present manifestations of this can generate $1 \mathrm{~cm}$ wide tapes that have $I_{c}(77 \mathrm{~K}, 0 \mathrm{~T})$ values of $\sim 200 \mathrm{~A}$ and $\mathrm{J}_{\mathrm{c}}$ values of $\sim 10^{6} \mathrm{~A} / \mathrm{cm}^{2}$ [27]. The great potential of this technique for making YBCO conductors has led to much current interest in implementing the result by other means. The crucial step in the IBAD process is the formation of the aligned YSZ layer, which is very slow, $<0.1 \mathrm{~nm} / \mathrm{sec}$. In fact some YSZ texturing can be obtained under specific growth conditions without the IBAD step. Such a process may be viable if a somewhat lower $\mathbf{J}_{c}$ can be tolerated ( few $\left.10^{5} \mathrm{~A} / \mathrm{cm}^{2}\right)$ [29]. 


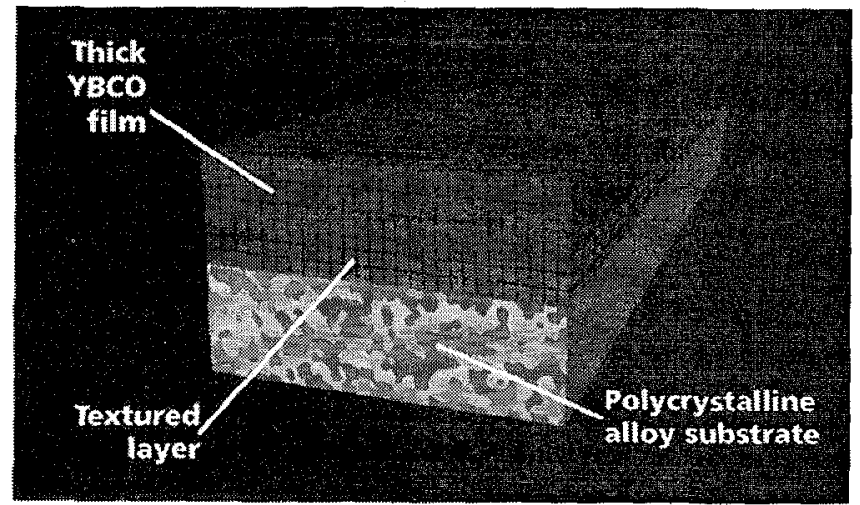

Figure 5: Schematic diagram of the formation of a coated conductor of YBCO, showing the textured intermediate buffer layer formed by ion beam assisted deposition (courtesy P. Arendt and S. Foltyn LANL).

The RABiTS process in principle offers a way around the IBAD step, for in this case thermo-mechanical processing of a (generally pure) $\mathrm{Ni}$ substrate tape can produce an almost single-crystal like texture in the Ni. This is suitable for, with certain easily applied buffer layers [28], growth of an epitaxial $\mathrm{YBCO}$ layer. At present, the best $\mathrm{J}_{\mathrm{c}}$ values are within about $80 \%$ of the best IBAD tapes.

Both of the above processes are important for the opportunity that they offer to fabricate conductors that offer $66-77 \mathrm{~K}$ critical current performance similar to that of $\mathrm{Nb}-\mathrm{Ti}$ at $4 \mathrm{~K}$. They also offer generic paths to the fabrication of any HTS conductor, for no other HTS compounds besides BSCCO have the self-aligning capability of the double Bi-O layer that is so vital to their high $J_{c}$ properties. It should be noted that, although YBCO is the main contender for biaxially aligned conductors, biaxial alignment makes conductors of Tl-1223 and the (Re-doped) Hg-cuprates [30] possible. Many papers on biaxially textured materials are being presented at this meeting. We may confidently expect that there will be many more at ASC98.

\section{WHAT REALLY IS J $\mathrm{J}_{\mathrm{c}}$ ?}

Although $J_{c}$ is the single most important parameter to be maximized for application, the term is often very loosely defined and what controls it is often not clear in any particular HTS conductor. In fact there are many ways of defining the critical current density and since they can differ by many orders of magnitude the potential for confusion is considerable. To clarify when taking a practical view, it is instructive to work back from the actual measurement. For large scale applications, the crucial measurement is that of the transport critical current, $I_{c t}$. This defines a transport current density $\mathrm{J}_{c t}=\mathrm{I}_{c t} / \mathrm{A}$, where $\mathrm{A}$ is the whole cross-sectional area of superconductor. Thus $\mathbf{J}_{c t}$ defined in this way is a macroscopic, not a local microscopic parameter. This takes $\mathbf{J}_{\mathrm{ct}}$ far from the actual physics which determines the local critical current density determined by flux pinning.

Much of the uncertainly about $\mathrm{J}_{c}$ is in fact uncertainty about A. For an engineer it is natural to take $A$ as the whole super- conductor cross-section (or perhaps more reasonably the whole conductor cross-section), but from the scientific point of view A needs to be defined as the local section carrying current. As noted in the following section, the local current densities defined by flux pinning, $J_{f p}$, can be very high. What the BSCCO wire fabricator is so far able to deliver may be only $10^{-3}$ or $10^{-4}$ of $\mathrm{J}_{\mathrm{fp}}$, for reasons that we now discuss (the discussion in the rest of this section is all made for BSCCO2223) [31].

\section{A. The depairing critical current density $J_{d}$}

The fundamental limit is the depairing current density, $J_{d}$ whose magnitude is given approximately by $J_{d} \sim H_{c} / \lambda$, where $\mathrm{H}_{\mathrm{c}}$ is the critical field $(0.3-1 \mathrm{~T})$ and $\lambda$ the penetration depth [32]. For BSCCO-2223, this gives $\mathbf{J}_{d}=1-3 \times 10^{8} \mathrm{~A} / \mathrm{cm}^{2}$ in the zero temperature limit, reduced by about an order of magnitude at $77 \mathrm{~K}$. Thus $J_{\mathrm{d}}$ is quite high enough to provide no fundamental limit to economic operation.

\section{B. The flux pinning critical current density $J_{j p}$}

A fundamental issue for any HTS compound is its electron mass anisotropy since this determines the range of reduced field $\left(\mathrm{H} / \mathrm{H}_{\mathrm{c} 2}\right)$ over which flux pinning can be effective. Unfortunately BSCCO-2223 is strongly anisotropic, leading to a strong suppression of the range of practical use, the irreversibility field, $\mathrm{H}^{*}(77 \mathrm{~K})$, being $0.2-0.3 \mathrm{~T}$. Below $\mathrm{H}^{*}$ current densities of 0.01-0.1 $J_{d}$ should be feasible, thus leading to $\mathrm{J}_{\mathrm{fp}} \sim 10^{6}-10^{7} \mathrm{~A} / \mathrm{cm}^{2}$ (at $0 \mathrm{~K}$ with no flux creep), derated by about an order of magnitude at $77 \mathrm{~K}$. These predictions are consistent with the $\mathrm{J}_{\mathrm{ct}}$ values measured on epitaxial BSCCO-2223 films. Two studies [33,34] report $\mathrm{J}_{\mathrm{c}}(77 \mathrm{~K}, 0 \mathrm{~T})$ greater than $10^{6} \mathrm{~A} / \mathrm{cm}^{2}\left(\mathrm{~T}_{\mathrm{c}} 105 \mathrm{~K}\right.$ and $\left.96-99 \mathrm{~K}\right)$ and one [35] greater than $10^{5} \mathrm{~A} / \mathrm{cm}^{2}\left(\mathrm{~T}_{\mathrm{c}} 92-97 \mathrm{~K}\right)$. These high $\mathrm{J}_{\mathrm{fg}}$ values are 10-100 times the threshold required for economic operation.

Polycrystalline conductor forms have much smaller $J_{c t}$, reproducible values falling into the range $10-50 \mathrm{kA} / \mathrm{cm}^{2}$, only $1-5 \%$ of the best thin film values. The most plausible rationalization is that polycrystals contain many weak links and other barriers to current flow. To deduce whether flux pinning plays much role in determining $J_{c t}$ we can study the irreversibility field $\mathrm{H}^{*}$. There is interesting detail to the way that $\mathrm{H}^{*}$ changes with the processing condition in $\mathrm{BSCCO}$ 2223 tapes, as shown by recent experiments of Edelman et al. [36] and Parrell et al. [37]. In contrast to the very high $J_{\text {c }}$ films cited above $[34,35]$ for which $\mathrm{H}^{*}$ was $<100 \mathrm{mT}$ at $77 \mathrm{~K}$, $\mathrm{H}^{*}$ for these much lower $\mathrm{J}_{\mathrm{c}}$ tapes is 2 to 3 times higher (200$300 \mathrm{mT}$ ). This anti-correlation is a strong hint that the large difference in magnitude of $\mathbf{J}_{c t}$ of tapes and films below $\mathrm{H}^{*}$ is more determined by their connectivity than by flux pinning. Nevertheless, $\mathrm{H}^{*}$ and $\mathrm{J}_{c}$ in tapes undergo systematic changes with the degree of reaction [36] and of the effective equilibration temperature of the 2223 phase [37], perhaps reflecting the influence of varying cation stoichiometry on flux pinning, A really striking dependence of $J_{c}$ (and also 


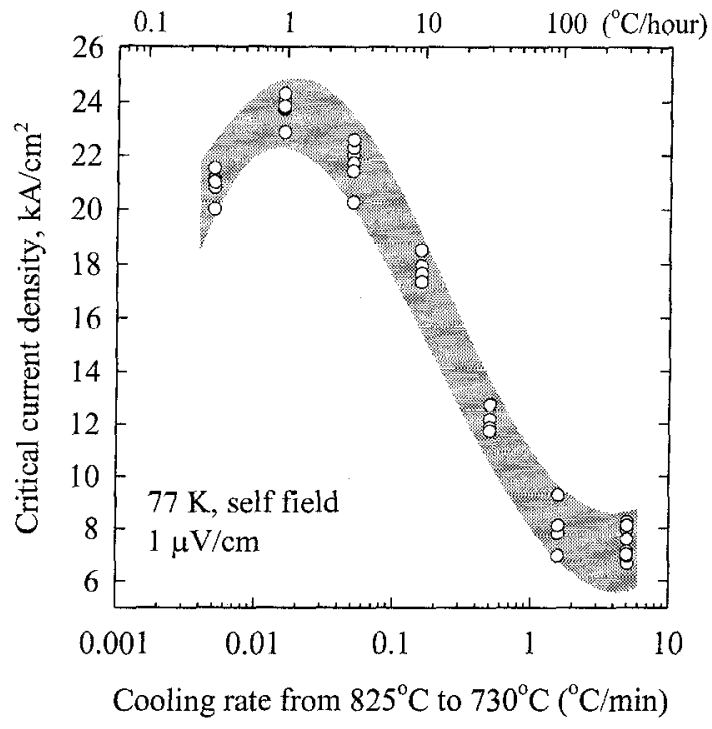

Figure 6: Transport $\mathrm{Jc}_{\mathrm{c}}(77 \mathrm{~K}, 0 \mathrm{~T})$ of a multifil amentary BSCCO-2223 composite of the design of Fig. 3 as a function of changes in the final cooling rate (Courtesy J. A. Parrell, ref. 36). The irreversibility field also increases monotonically with the increase of $J_{c}$

$\mathrm{H}^{*}$ ) on cooling rate after final heat treatment is shown in Figure 6.

\section{The intergranular critical current density $J_{b}$}

The way that GBs impede current flow in BSCCO tapes has been much debated $[38,39]$, though there is little single GB data from bicrystals (and this is all on BSCCO-2212) [40-43] that leads to definitive conclusions. Recent bicrystal data from our group on both YBCO and BSCCO-2212 is summarized in [44]. A key issue is which GBs can be in the current path without degrading $J_{0}$. V-I curves measured on BSCCO-2212 bicrystals of [100] and [001] tilt show less fall off with misorientation angle than for thin film YBCO [001] tilt bicrystals, implying that low angle "Railway Switches" are acceptable. However, when the grain boundaries become basal-plane faced, there appears a quasi-ohmic dissipation at low voltages that we interpret as being the signature of $c$-axis current flow, which is both more dissipative and qualitatively different (e.g. it does not have a definable $\mathrm{H}^{*}$ ) from the $\mathrm{ab}$ plane flow seen in [001] and [100] tilt grain boundaries. Thus basal-plane faced boundaries appear to be deleterious to the local $J_{c}$, even though they may still be vital to permit percolation along long lengths of conductor.

\section{Percolation current density $J_{p}$ of a polycrystalline tape} Grain boundaries are only one barrier to current flow through the polycrystalline network. Additional, more macroscopic barriers are unconverted non-superconducting phases, cracks, pores and section irregularities due to filament sausaging. All of these defects reduce the cross-section carrying transport current. Thus it is easy to see that the magnitude of $J_{c}$ defined by $\mathrm{I}_{\mathrm{c}} / \mathrm{A}$ can be much less than the flux pinning limit. Because it seems likely that less than $10 \%$ of the cross-

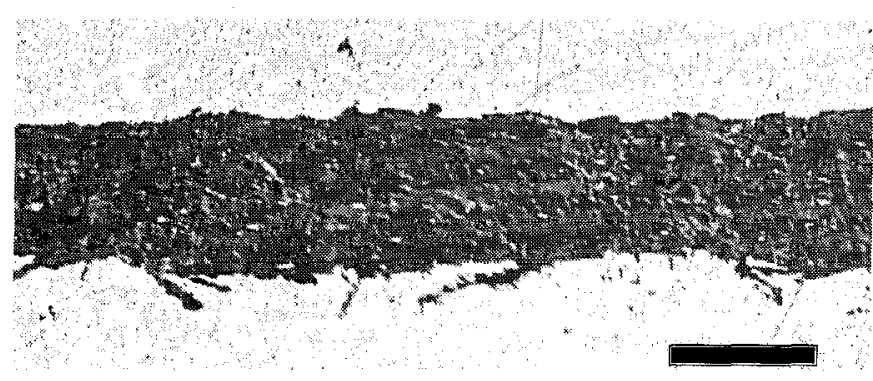

$\mathbf{5 0} \mu \mathrm{m}$

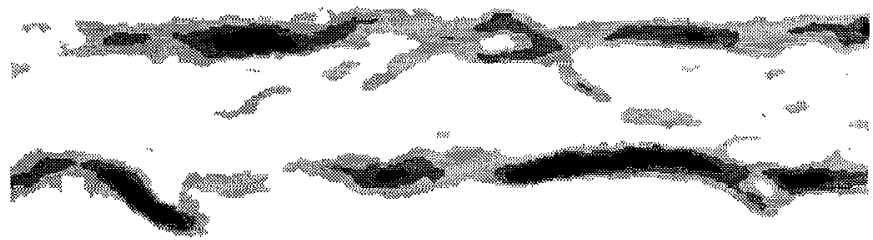

Figure 7: Polarized light microstructural image (upper) and 2D contour map (lower) of the local current density reconstructed from the magneto-optical image of a BSCCO-2223 filament taken in a field of $44 \mathrm{mT}$ at $10 \mathrm{~K}$. The 3 shades of gray correspond to local current densities $\mathrm{J}_{\mathrm{c}}$ : lightest $1.6-2.6 \times 10^{5}$, $2.6-3.6 \times 10^{5},>3.6 \times 10^{5} \mathrm{~A} / \mathrm{cm}^{2}$. Current flow preferentially near the AgBSCCO interface and along larger, better aligned grains. The percolative nature of the current flow is quite obvious. (Courtesy A. Pashitski, ref. 45).

section of typical tapes is carrying current effectively, it becomes important to analyze the possible role of all defects that appear in tapes. Recently, magneto-optical imaging has been applied to this task [45-46], as shown in Fig. 7. Data such as this directly show that the percolative path through the network is the prime variable controlling the magnitude of $\mathrm{J}_{\mathrm{c}}$, not $\mathrm{J}_{\mathrm{d}}, \mathrm{J}_{\mathrm{fp}}$, or $\mathrm{J}_{\mathrm{b}}$. This insight is vital in the drive to strongly enhance $\mathrm{J}_{\mathrm{c}}$.

\section{E. The role of cracks in controlling the percolation current} density $J_{p}$ of tapes

An important hypothesis is that cracks are a major barrier to current flow in BSCCO tapes. To test this hypothesis explicitly is not easy but recent experiments in our group [47-48] used magneto-optical imaging to directly demonstrate the important role that cracks can play. Partial results are shown in Fig. 8, where rolled and pressed samples otherwise identically treated) after the third and final heat treatment are contrasted. The pressed sample exhibits strong flux exclusion and a high $J_{c}\left(20 \mathrm{kA} / \mathrm{cm}^{2}\right)$, while the rolled sample is very much more non-uniform and has only some $20 \%$ of the $J_{c}$ in the rolled tape. This contrast between rolled and pressed tape is common. One reason that cracks are hard to avoid at final stages is that the liquid that eases their healing is used up in reacting to form the 2223 phase. This area of process control [49] seems to be a very fertile one in raising $J_{c}$.

\section{COSTS}

One sign of the success of HTS conductors is that the cost of making serious devices is now becoming an issue. At present there is little concrete that can be said about costs 
except to say that the DOE target price of $\sim \$ 10 / \mathrm{kA} . \mathrm{m}$, based on $77 \mathrm{~K}, 0 \mathrm{~T}$ performance, needs to be taken seriously if HTS conductors are to stake out serious markets. There seems to be no reason why this is not possible, since the raw material costs of HTS and LTS conductors are not greatly different. What really will determine the costs is the depth of our materials understanding, for this determines the $J_{c}$ component of the cost. Although the complexity of HTS materials science is sometimes daunting, there is a large headroom between what they are capable of and what many applications need. My personal feelings are optimistic, not least because I believe in the broad base of scientific understanding that is now being generated. Good collaboration between users, manufacturers and researchers can have enormous payoffs here, as exemplified by the Wire Development Group, one of whose recent $\mathrm{J}_{\mathrm{c}}$ successes is reported at this meeting [22].

\section{WTEC/ JTEC PANEL ON POWER APPLICATIONS}

An important and useful resource for assessing future energy applications of superconductivity is the forthcoming report by the World Technology Development Center (WTEC) [50] of the comparative strengths of programs in the US, Japan and Germany as of mid-1996. Some of the key points of this study are that the expectation for HTS programs in Japan and Germany are more long term. Thus government support in Japan and Germany for what are still seen as largely, pre-competitive programs is rather secure in the near term. Both countries see superconductivity as a major $21^{\text {st }}$ century technology. By contrast there is a shorter term focus in the US. Partly this is a consequence of the more short-term nature of federal budget commitments in the US, and partly due to political debate about the role of government in technology development exercises. Another major
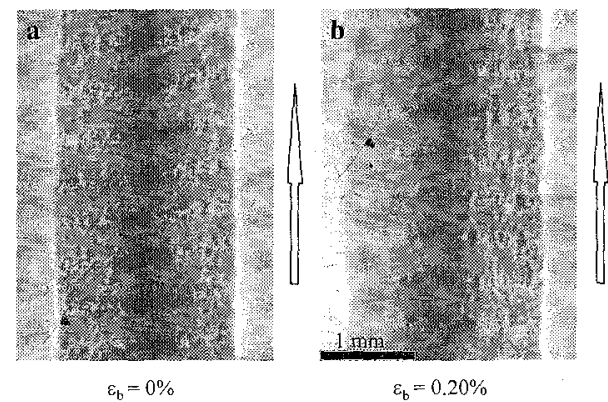

d.

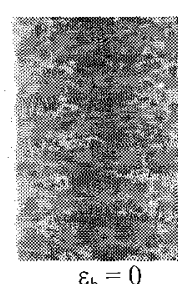
$\varepsilon_{\mathrm{b}}=0.20 \%$
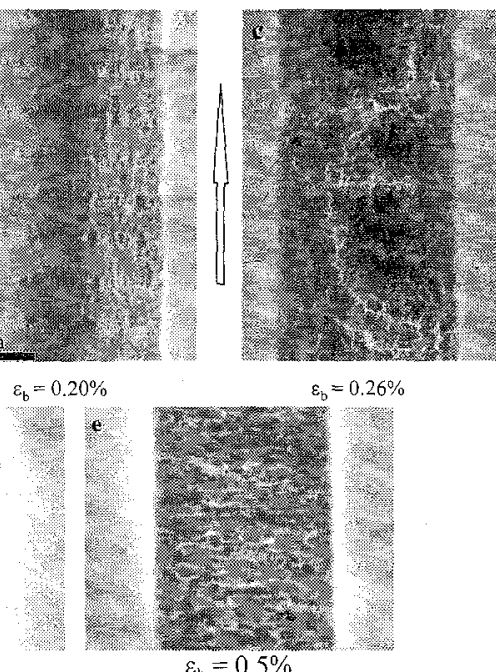

$\varepsilon_{\mathrm{b}}=0.26 \%$

Figure 8. Plan-view magneto-optical images of (a) a pressed sample, (b) a pressed sample bent to a strain of $0.2 \%$, (c) a pressed sample strained to $0.26 \%$, (d) a rolled sample, and (e) a rolled sample strained to $0.5 \%$. Cracks (networked bright areas) propagate along the tape axis in pressed samples, and transverse to the axis in rolled samples. (Courtesy J. Parrell, A. Pashitski, A. Polyanskii and M. Polak (UW). difference is that small companies, particularly those funded by venture capital, have driven rapid US progress in the field. The natural desire of such companies to seek a more immediate payoff than that demanded by the multi-billion dollar companies that dominate Japanese and German work adds a distinctively different flavor to US programs. As a whole, this makes US and German and Japanese programs complementary, not just competitive.

To emphasize continuity, I would like to close this section with a quote from John Hulm's 1987 National Academy Report: "The short term problems and long term opportunities (of HTS) are both easily underestimated...The science and technology (of HTS) are strongly intertwined, requiring simultaneous progress in basic science, processing science, and applications....(This is a field in which we need to) take a long term, multidisciplinary view..... The pre-commercial applications of HTS to new markets will extend for a decade or more."

These few sentences still appear as relevant today as when they were written 9 years ago.

\section{TECHNICAL SUMMARY}

It is salutary to remember that, for all the enthusiasm of early 1987, it appeared by year's end that there was a "fatal flaw", the flaw being the curse of poorly performing grain boundaries. But fortunately, believers in HTS did not give up and the breakthrough needed to make conductors was demonstrated in 1989. Kilometer-length, useful BSCCO conductors are now available from several companies world wide. Steady progress, largely empirical at first but now significantly science-driven, is bringing working prototypes of power cables, fault current limiters, motors, transformers, and other components of an HTS technology, in most cases accessing technologies complementary, rather than competitive to LTS applications. Second generation, higher field, higher temperature $\mathrm{YBCO}$ conductors are being developed and may transform future ASC conferences. Progress in making such conductors is very much gated by the need to understand how current travels in HTS conductors and how to control their complex materials science. The complexity of the HTS cuprates cannot be lightly underestimated but fortunately their underlying properties give us plenty of headroom in $J_{c}$. There are many new applications for HTS power devices not served by LTS. Given that we have had just 7 years, not 10 , to flesh out the dreams of 1987, it seems fair to predict a bright technical future for large scale applications of HTS!

\section{A SOCIOLOGICAL AND POLITICAL AFTERWORD}

Unfortunately one cannot just end with the scientific and the technical. Support for the application dreams of HTS also requires a public belief in their future and this support is no longer as unquestioned as it used to be. Each of us is there- 
fore called upon to educate the public, our families and friends, not just our technical colleagues, as to why the applications of superconductivity are a benefit to society. In short we need a vision for the public, not just ourselves. The great complexity of HTS materials also dictates collaboration, for no group is big enough or good enough to solve all important issues. Progress in the field is world-wide, as attendance at this meeting demonstrates. Thus success anywhere is success for the field and opportunity for all! The implications of all of this are surely positive for this still exciting field, providing that we adjust our work intelligently to the changing demands of society!

\section{ACKNOWLEDGEMENT}

To cover such a vast field, I have benefited from many discussions with colleagues through out the world. In Madison, I have benefited particularly from my own group members, as well as those in Eric Hellstrom's and Susan Babcock's groups. Colleagues at ANL, ASC, LANL, NIST, ORNL who are participants in the Wire Development Group have been particularly helpful in helping develop an integrated view of BSCCO-2223. Our hosts in Japan, Germany and Switzerland during the summer $1996 \mathrm{JTEC} / W T E C$ exercise are also acknowledged for their gracious sharing of information on recent and hoped-for future advances in this field. I am also very grateful to colleagues at $\mathrm{LBL}, \mathrm{FNAL}, \mathrm{BNL}$ and in the LTS wire industry who have participated in the Nb-Ti Workshop over many years and who have shown there too the great benefits of collaborating and competing in a coordinated way. I note also my gratitude for support for different aspects of this work from DARPA, DOE, EPRI, ONR and NSF.

\section{REFERENCES}

[1] IEEE Trans. on Magnetics, vols. 23, 25, 27, 1987, 1989, 1991 and IEEE Trans. on Appl. Superconductivity, vols. 3, 5, 1993, 1995.

[2] G. Yntema IEEE Trans. on Magnetics, vol. 23, p 3901987.

[3] T. G. Berlincourt, IEEE Trans. on Magnetics, vol. 23, p 4031987

[4] J. N. Rjabinin and L.V. Shubnikov, Nature vol. 135, p. 5811935.

[5] A. A. Abrikosov, Sov. Phys. JETP, vol. 5, p. 1174, 1953

[6] D. Dew Hughes, Cryogenics, vol. 36, p. 660, 1986.

[7] J. G. Bednorz and K. A. Muller, Z. Physik, vol. B64, 1986, p. 189.

[8] M.K. Wu et al. Phys. Rev. Lett. vol. 58, p 908, 1987.

[9] H. K. Onnes, Comm. of Physic. Lab. Leiden, Suppl..34b to vol. 133144,1913, pp. 37.

[10] J. Hulm, Research Briefing on High Temperature Superconductivity, National Academy of Sciences, NAS Press 1987.

[11] "Superconductivity: Challenge for the Future", Federal Conference on Commercial Applications of Superconductivity, July 28-29, 1987.

[12] A. Narath, Panel on High $-T_{c}$ Superconducting Magnet Applications in Particle Physics, DOE/ER-0358, Department of Energy, December 1987.

[13] P. Grant, "Superconductivity and Electric Power: Promises, Promises...Past, Present and Future.", to appear, IEEE Trans. on Appl. Superconductivity, vol, 7, 1997.

[14] C. W. Chu, "Materials Challenges and Opportunities: The next ten year", ibid ref. 13.

[15] T. Van Duzer, "Superconductor Electronics", ibid ref. 13.
[16] K. Heine, N. Tenbrink, M. Thoener, Appl. Phys. Lett. vol. 55, 1989, p. 2441.

[17] P. J. Lee, D. C. Larbalestier, IEEE Trans. on Appl. Superconductivity, vol. 3, 1993 p. 833.

[18] The Low Temperature Superconductor Workshops have been held continuously since 1983 , alternately hosted by D. C. Larbalestier (U. of Wisconsin) and by R. M. Scanlan (Lawrence Berkeley Laboratory).

[19] See for example papers in IEEE Trans. on Appl. Superconductivity, vol. 5, 1995, pp. 298-306.

[20] L. D. Cooley, P. J. Lee, D. C. Larbalestier, Phys. Rev. B, vol.53, p.6638, 1996.

[21] K. Sato et al., "Large scale applications using BSCCO conductor", ibid ref. 13.

[22] Q. Li et al. "Progress towards practical Bi-2223 conductors," ibid ref. 13.

[23] M. Suenaga, D. O. Welch, R. Budhani, Sup. Sci. and Tech. vol. 5, p. $\mathrm{S} 1,1992$.

[24] The Wire Development Group is a collaboration between groups at American Superconductor Corp., Argonne National Lab., Los Alamos National Lab., the National Institute of Standards and Technology (Gaithersburg), Oak Ridge National Lab., and the University of Wisconsin.

[25] R. Flukiger et al., Inst. Of Physics Conf. Proc. \#148, p. 57, 1995

[26] Y. Iijima, N. Tanabe, O. Kohno, and Y. Ikeno, Appl. Phys. Lett. Vol. 60, p.769 1992

[27] X. Wu et al., Appl. Phys. Lett. Vol. 67, p. 23971995.

[28] D.P. Norton, et al., Science, vol. 274, p. 755,1996.

[29] K. Fujino et al., Adv. In Superconductivity V'III, p. 675, 1995.

[30] J. Shimoyama, et al., Adv. In Superconductivity VII, p. 287, 1994

[31] D. C. Larbalestier, Proc. of the $10^{\text {th }}$ Anniversary HTS workshop on Physics, Materials and Applications, Ed. B. Batlogg, World Scientific, Singapore, p. 41, 1996.

[32] Qiang Li, M. Suenaga, T. Hikata, K. Sato. Phys. Rev, vol, B46 (1992) 5957.

[33] Y. Hakuraku and X. Mori, J. Appl. Phys. 73 (1992) 309

[34] P. Wagner, X. Frey, X. Hillmer, X. Adrian, Phys. Rev. B51 (1995) 1206.

[35] H. Yamasaki et al. IEEE Trans. on Superconductivity. 3 (1993) 1536.

[36] J. A. Parrell, D. C. Larbalestier, G. N. Riley, Q. Li, R. Parella, M. Teplitsky, Appl. Phys. Lett. , vol. 69, p. 2915, 1996.

[37] H. S. Edelman, J. A. Parrell, D. C. Larbalestier, J. Appl. Phys, vol. 81, p. $2296,1997$.

[38] L. N. Bulaevskii, J. R. Clem, L. I. Glazman, and A. P. Malozemoff, Phys. Rev. vol. B45, p. 2545, 1992.

[39] B. Hensel, J.-C. Grivel, A. Jeremie, A. Perin, A. Pollini, and R. Flukiger, Physica C, vol. 205 p. 329, 1993.

[40] N. Tomita, Y. Takahashi, M. Mori, and Y. Ishida, Jpn. J. Appl. Phys. Vol. 31, p L942, 1992.

[41] J-L. Wang, X.Y. Cai, R.J. Kelley, M.D. Vaudin, S.E. Babcock, and D.C. Larbalestier, Physica C, vol. 230, 189, 1994

[42] J-L. Wang, X.Y. Cai, R.J. Kelley, M.D. Vaudin, S.E. Babcock, and D.C. Larbalestier, J. Mater. Res. vol. 11, p. 868, 1996.

[43] Qiang Li et al.,. Appl. Phys. Lett., vol. 70, p. 1164, 1997.

[44] D. C. Larbalestier et al., , Proceedings of 8th International Conference on Critical Currents of Superconductors, Kitakyushu, Japan, Ed. K. Yamafuji, World Scientific 1996, p. 87, 1996

[45] A. Pashitski, A, Polyanskii, A. Gurevich, J. A. Parrell, D. C. Larbalestier, Physica C vol. 246, p. 133, 1995.

[46] U. Welp et al., Appl. Phys. Lett, vol. 66, p. 1270, 1995.

[47] J. A. Parrell, A. Polyanskii, A. Pashitski, D. C. Larbalestier, Super. Sci. and Tech, vol. 9, p. 393, 1996

[48] J. A. Parrell, S. E. Dorris, D. C. Larbalestier, J. Mater. Res., vol. 11, p. 555,1996

[49] M. Polak et al., Appl. Phys. Lett. vol. 70, p. 1034, 1997.

[50] D. C. Larbalestier et al., "Power Applications of Superconductivity in Japan and Germany", Report of the JTEC/WTEC Panel, International Technology Research Institute, Loyola U., Baltimore MD, 1997. 